\title{
Ranking the acute poisoning etiologies in Iran: A systematic review and meta-analysis
}

\section{Saeedeh Derhami ${ }^{1}$, Ehsan Bolvardi², Reza Akhavan², Mahdi Foroughian², Behzad Shahi ${ }^{3}$, Arman Hakemi ${ }^{1}$, Zhila Rahmanian", Samaneh Abiri ${ }^{4 *}$}

'Department of Emergency Medicine, Mashhad University of Medical sciences, Mashhad, Iran

2Department of Emergency Medicine, Faculty of Medicine, Mashhad University of Medical sciences, Mashhad, Iran ${ }^{3}$ Department of Emergency Medicine, Faculty of Medicine, Zahedan University of Medical sciences, Zahedan, Iran

${ }^{4}$ Department of Internal Medicine, Jahrom University of Medical sciences, Jahrom, Iran

${ }^{5}$ Department of Emergency Medicine, Jahrom University of Medical Sciences, Jahrom, Iran

\begin{abstract}
Received: 17 August 2020
Accepted: 11 October 2020

Published online: 22 October 2020

*Corresponding author:

Samaneh Abiri; Department of

Emergency Medicine, Jahrom

University of Medical sciences,

Jahrom, Iran.

Email: Samaneh.abiri@gmail.com

Competing interests: None.

Funding information: None

Citation: Derhami S, Bolvardi E,

Akhavan R, Foroughian M, Shahi

B, Hakemi A, Rahmanian Z, Abiri

S. Ranking the acute poisoning

etiologies in Iran: A systematic

review and meta-analysis. Journal

of Emergency Practice and Trauma

2021; 7(2): 82-87. doi: 10.34172/

jept. 2020.40
\end{abstract}

\begin{abstract}
Objective: Acute poisoning is a major health problem and one of the most common causes of emergency visits worldwide. Since most poisoning subjects present with a decreased level of consciousness and due to unreliable disease history, recognizing the etiological cause of the poisoning represents a critical part in arranging the treatment strategy. This study aimed at examining the prevalence of etiological causes of poisoning in Iran in a systematic review and meta-analysis.

Method: This systematic review and meta-analysis investigated the cross-sectional studies published from 1990 to 2020, reporting specific poisoning agents among acute poisoning cases in Iran. Persian and English articles on this subject were collected by searching the Scientific Information Database (SID), ScienceDirect, PubMed, Medlib, IranMedex, Scopus, Magiran, and Google Scholar databases. The heterogeneity of the studies was investigated using the 12 index and the probability of bias in the publication was assessed by the Begg and Mazumdar test with a significance level of 0.1. Data analysis was performed by Comprehensive Meta-analysis software version 3 (Biostat, Englewood, NJ, USA).

Results: In our review, 19 studies appraising 143,251 cases of poisoning were included The ranking of the OR of each agent was done; Opium poisoning was the most prevalent poisoning case followed by benzodiazepine, acetaminophen, antipsychotic medications, organophosphates, aluminum phosphide, amphetamine, pesticide, tricyclic antidepressant (TCA), alcohol, chemicals, carbon monoxide (CO), nonsteroidal anti-inflammatory drugs (NSAIDs), and selective serotonin reuptake inhibitors (SSRIs).

Conclusion: While proper judgment on the cause of poisoning and selection of suitable treatment manners could be followed by a very good prognosis in patients with acute poisoning; this demands an epidemiological perception of the prevalence of the etiological poisoning agents. Our study ranked the most likely agents leading to the poisoning, to be at the top of the list of differential diagnoses of physicians.

Keywords: Acute poisoning, Iran, Meta-analysis
\end{abstract}

Introduction

Decreased level of consciousness can be an acute and potentially life-threatening state that necessitates prompt management to sustain life and restore normal brain functions (1). One of the most important skills that emergency physicians should have is how to manage patients with an altered level of consciousness (ALC) (2). Having a clear diagnostic and treatment algorithm in the face of these patients is vital to save their lives $(3,4)$. The non-traumatic decreased level of consciousness is an important emergency due to the high mortality rate if not properly managed (5). Decreased levels of consciousness can be started with drowsiness or get progressed to stupor (severe drowsiness) or coma. In a coma, the patient is unable to communicate verbally so there would not be a reliable history of the disease. Data on the prevalence of common causes of ALC in each region can help diagnose the etiology of the disease (6). The etiology of decreased level of consciousness plays an important role in predicting the prognosis (7). Poisoning is one of the most serious etiologies to reduce the level of consciousness. Also, unknown drug toxicities may contribute to a decreased level of consciousness with an urgent need for early diagnosis, treatment, and special care. In examining the causes of decreased consciousness and coma, major etiologies include poisoning and 
metabolic disorders (8). Finding the poisoning etiology is the most important part of ALC management, which should be conducted through a precise history taking and performing accurate clinical examinations (9). However, there may not be much information in the patient's history with a decreased level of consciousness. The toxin is a substance that, if ingested in sufficient amounts, can interfere with health and lead to temporary or permanent damages to the body. Toxins may be solid, liquid, or gaseous. Toxins cause adverse effects after entering the body, which is called poisoning. The signs and symptoms of poisoning vary depending on the type of toxin and the way it enters, and they appear quickly or over several days (10). Due to the different treatment methods and antidotes for different toxins, it is very important to diagnose the type of poisoning (11). Opioids, medications, Rice pill (aluminum phosphate) and various toxins that may be mistakenly or intentionally used for suicide are common causes of poisoning. To prevent the potential dangers of poisoning, epidemiological information is of particular importance and the epidemiological pattern of poisonings on a national scale should be examined to give a clear view to physicians.

\section{Methods}

This systematic review and meta-analysis was undertaken to investigate the prevalence of poisoning etiology in Iran based on the Preferred Reporting Items for Systematic Reviews and Meta-Analyses (PRISMA) statement.

The search strategy embedded in this study was based on both English and Persian researches conducted in Iran. Scientific information databases of Jihad Daneshgahi University (SID), Medlib, IranMedex, Google scholar, and Magiran for Persian studies, and PubMed, ScienceDirect, and Scopus for articles in English were queried based on the various combinations (AND/OR) of Medical Subject Headings (MeSh) keywords [acute poisoning, Iran, "Agent" poisoning, Drug Overdose]. The query was limited to peer reviewed English or Persian language studies, mainly conducted in Iran. Two researchers independently selected eligible studies and in case of incompatibility in each step of the study, a third researcher was negotiated to make the final decision. The inclusion criteria for studies were (1) the cross-sectional studies reporting etiology of the poisoning in acute poisoning cases having the ICD10 criteria of the poisoning definition; (2) quality assurance based on the STROBE checklist statement (Table S1). STROBE Statement-checklist of items that should be included in reports of observational studies, based on the latest version (https://www.strobe-statement.org), had 32 items and studies which had at least 29 items were included in study. Item of "Describing any efforts to address potential sources of bias" was considered indispensable.

Initially, all articles related to poisoning in Iran were collected and after the search, a list of abstracts was prepared by the researchers and duplicates were removed $(n=31)$. At this stage, all the articles in which the prevalence of poisoning was mentioned in the abstract were included in the initial list for full-text evaluation. A checklist was used to assess the inclusion criteria of our study and the data collection (including items of researcher's name, title, year of publication, city, sampling method, sampling number, study type, total prevalence, and toxicology etiology). Based on these steps, 185 articles were detected in the primary search, and 78 articles with related title and abstract were included in full-text evaluation. In addition, the reference lists of all selected articles were screened for potentially relevant publications. Finally, 19 eligible articles were entered into the meta-analysis stage (as shown in Figure 1). The ratio of each poisoning

Table 1. Included studies in the meta-analysis

\begin{tabular}{|c|c|c|c|c|}
\hline Author, year & Female: Male (n/\%) & Number of cases & Death rate & Mean age \\
\hline Sadeghieh Ahari, 2012 & $66.1 \%: 33.9 \%$ & 245 & $33.5 \%$ & 30.52 \\
\hline Torkashvand, 2015 & $45.4 \%: 54.6 \%$ & 260 & $1.90 \%$ & $23.10 \pm 15.16$ \\
\hline Najari, 2017 & $16: 39$ & 7732 & $0.9 \%$ & $49.84 \pm 20.28$ \\
\hline Dehghani, 2015 & $47.6 \%: 52.4 \%$ & 163 & $2.4 \%$ & NA \\
\hline Hashmnejad, 2014 & 102: 102 & 204 & $5.8 \%$ & $29.8 \pm 13.4$ \\
\hline Ahmadi, 2011 & NA & 2057 & $1.3 \%$ & NA \\
\hline Afzali, 2008 & 69: 349 & 418 & $3.6 \%$ & 40.5 \\
\hline Masoumi, 2013 & $95.1 \%: 4.9 \%$ & 402 & $2.0 \%$ & $19-44$ \\
\hline Eslami, 2014 & $34.9 \%: 65.1 \%$ & 988 & $2.8 \%$ & NA \\
\hline Jafarzadeh, 2017 & $36.72 \%: 63.28 \%$ & 275 & $0 \%$ & $28.20 \pm 18.60$ \\
\hline Feiz Disfani, 2019 & 130: 113 & 243 & $3.8 \%$ & Under 15 years \\
\hline Shokrzadeh, 2017 & $45.9 \%: 54.1 \%$ & 800 & $1.6 \%$ & $19.53 \pm 14.39$ \\
\hline Sobhani, 2000 & $51.4 \%: 48.6 \%$ & 1215 & $1.06 \%$ & Under 15 years \\
\hline Islambulchilar, 2009 & $55.7 \%: 44.3 \%$ & 1342 & $2.3 \%$ & $26.83 \pm 12.59$ \\
\hline Abdollahi, 1997 & $\approx 4500: 2500$ & 7000 & NA & NA \\
\hline Vatandoost, 2002 & 12233: 7278 & 19511 & NA & $>13$ years \\
\hline Afshari, 2004 & $53.4 \%: 46.6 \%$ & 71589 & 22.3 & $22.3 \pm 14.38$ \\
\hline Gorgi, 2016 & 89:103 & 17,342 & NA & NA \\
\hline Hassanian-Moghaddam, 2008 & 5583: 5818:64 unknown & 11,465 & $1 \%$ & $>12$ years \\
\hline
\end{tabular}




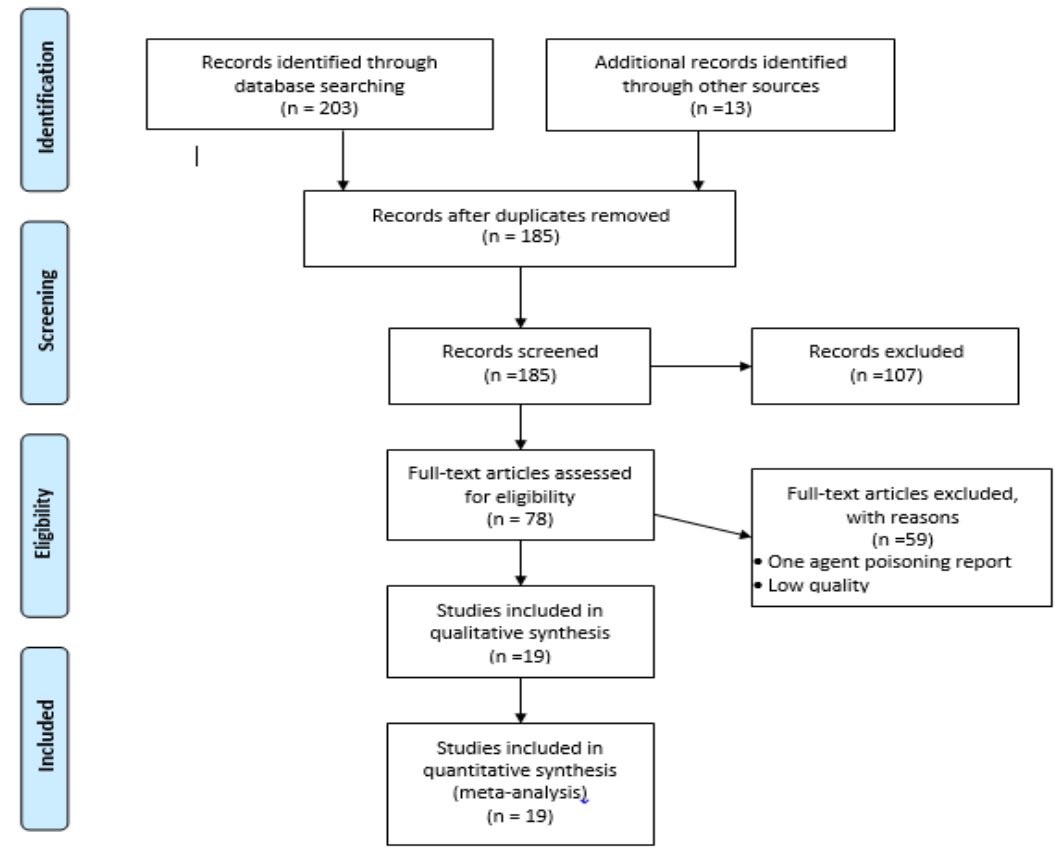

Figure 1. Study search strategy (PRISMA flow diagram).

agent to all of the poisoning cases was used to calculate the odds ratio (OR). The variance of each study was calculated according to the fixed-effect model. Studies were combined according to the number of samples and variance. Due to the heterogeneity of the studies, random effects were used to combine the studies. Cochran's test was used to evaluate the heterogeneity of studies using the I2 index. Begg and Mazumdar test revealed no possibility of the publication bias for each poisoning agent analysis $(P>0.05)$.

\section{Results}

As shown in Table 1, in our review, 19 studies evaluating 143,251 cases of poisoning were included (12-30). The age of most subjects was between 12-45 years old. In 8 studies, the number of female poisoning cases was dominant. The average death rate was $5.39 \%$ (range: $0 \%-33.5 \%$ ).

To evaluate the incidence rate of the poisoning by each agent, a meta-analysis of studies reporting the number of poisoning by a specific agent from a whole poisoned subject population was conducted.

As shown in Table 2, five studies reported the prevalence of acetaminophen poisoning. Among 10985 evaluated cases, acetaminophen poisoning was responsible for 1043 cases $(9.49 \%)$. Pooled incidence OR for acetaminophen poisoning was 0.164 (95\% CI: $0.155-0.174 ; P=0.001$, $\left.\mathrm{I}^{2}=99.67 \%\right)$. The total incidence of alcohol poisoning was 698 cases in 16413 poisonings (4.25\%) with pooled OR of 0.054 (95\% CI: 0.051-0.058; $P=0.001, \mathrm{I}^{2}=98.00 \%$ ). Also, incidence of aluminum phosphide poisoning was $10.9 \%(984 / 8950)$ with polled OR of $0.115(95 \%$ CI: $\left.0.109-0.122 ; P=0.001, \mathrm{I}^{2}=95.09 \%\right)$. Incidence of amphetamine poisoning was $8.4 \%(831 / 9908)$ with polled
OR of 0.091 (95\% CI: 0.085-0.097; $P=0.001, \mathrm{I}^{2}=98.05 \%$ ). Besides, incidence of antipsychotic poisoning was $10.9 \%$ (1232/11241) with polled OR of 0.139 (95\% CI: 0.130 $\left.0.149 ; P=0.001, \mathrm{I}^{2}=99.06 \%\right)$. In addition, incidence of benzodiazepine poisoning was $19.9 \%$ (6312/ 31692) with polled OR of 0.204 (95\% CI: 0.200-0.209; $P=0.001$, $\left.\mathrm{I}^{2}=98.92 \%\right)$. Among 12401 evaluated cases, chemical poisoning was responsible for 383 cases (3.1\%). Pooled incidence OR for chemical poisoning was 0.033 (95\% CI: $\left.0.030-0.037 ; P=0.001, I^{2}=96.61 \%\right)$. Incidence of carbon monoxide (CO) poisoning was $1.8 \%(375 / 20648)$ with polled OR of 0.027 (95\% CI: 0.024-0.029; $P=0.001$, $\left.I^{2}=98.5 \%\right)$. Nonsteroidal anti-inflammatory drugs (NSAIDs), opium, pesticides, selective serotonin reuptake inhibitor (SSRI), organophosphate and tricyclic antidepressant (TCA) poisoning incidences were 1.7\% (40/2261), 25.1\% (4960/19794), 6.7\% (1894/27943), $1.3 \%(33 / 2475), 12.7 \%$ (690/5426), 5.7\% (174/3045), respectively. The Forrest plots of the meta-analysis are

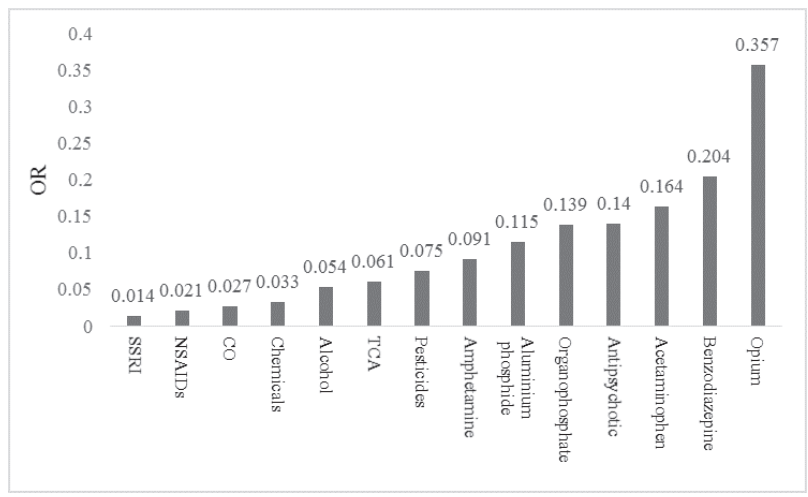

Figure 2. Ranking of each poisoning agent OR. 
Table 2. Meta-analysis of poisoning ratio based on the agent

\begin{tabular}{|c|c|c|c|c|c|}
\hline \multirow{2}{*}{ Agent } & \multirow{2}{*}{ Number of studies } & \multirow{2}{*}{ OR } & \multicolumn{2}{|c|}{$95 \% \mathrm{Cl}$} & \multirow{2}{*}{$I^{2}(\%)$} \\
\hline & & & Lower limit & Upper limit & \\
\hline Acetaminophen & 5 & 0.164 & 0.155 & 0.174 & 99.670 \\
\hline Alcohol & 6 & 0.054 & 0.051 & 0.058 & 98.000 \\
\hline Aluminum phosphide & 3 & 0.115 & 0.109 & 0.122 & 95.090 \\
\hline Amphetamine & 3 & 0.091 & 0.085 & 0.097 & 98.050 \\
\hline Antipsychotic & 7 & 0.140 & 0.133 & 0.148 & 99.060 \\
\hline Benzodiazepine & 8 & 0.204 & 0.200 & 0.209 & 98.920 \\
\hline Chemicals & 4 & 0.033 & 0.030 & 0.037 & 96.610 \\
\hline $\mathrm{CO}$ & 5 & 0.027 & 0.024 & 0.029 & 98.500 \\
\hline NSAIDs & 2 & 0.021 & 0.015 & 0.028 & 94.530 \\
\hline Opium & 10 & 0.357 & 0.348 & 0.365 & 99.720 \\
\hline Organophosphate & 4 & 0.139 & 0.130 & 0.149 & 97.800 \\
\hline Pesticides & 6 & 0.075 & 0.072 & 0.078 & 99.330 \\
\hline SSRIs & 2 & 0.014 & 0.010 & 0.019 & $28.02 \%$ \\
\hline TCAs & 2 & 0.061 & 0.053 & 0.070 & 95.980 \\
\hline
\end{tabular}

Abbreviations: CO, Carbon monoxide; SSRIs, selective serotonin reuptake inhibitors; TCAs, Tricyclic antidepressants; NSAIDs, nonsteroidal anti-inflammatory drugs.

provided in Supplementary file 1.

As shown in Figure 2, the ranking OR of each agent was done; Opium poisoning was the most prevalent poisoning followed by the benzodiazepine, acetaminophen, antipsychotic medications, organophosphates, aluminium phosphide, amphetamine, pesticide, TCA, alcohol, chemicals, CO, NSAIDs, and SSRIs.

\section{Discussion}

Due to the increasing use of medications in recent years, drug poisoning is a common cause of the level of consciousness (LOC) and in most cases can be treated with timely diagnosis and prompt treatment. Poisoning is one of the most common causes of emergency department visits, which can cause serious injuries and even death (31).

Acute poisoning refers to exposure to a toxic substance accidentally or over a short period, which can be intentional or unintentional (3). Unintentional or accidental poisoning is common and can lead to increased complications and mortality $(2,3)$. Most acute poisonings referred to the hospital are intentional poisonings that, regardless of their intentions and motives, potentially threaten a person's life and sometimes lead to death. Awareness of the pattern of poisoning in a particular area will play an important role in identifying risk factors and early detection of poisoning (2-4).

Due to this, the present study investigated the prevalence of etiological causes of poisoning in Iran in a systematic review and meta-analysis. We found out that opium poisoning was the most prevalent poisoning in Iran. In a study by Haghgou (32), it was stated that the pattern of intentional poisoning for suicide has changed from opium to pharmaceutical pills and chemical pesticides (19691974). While our analysis from 1997 to 2019 revealed opium as the most common cause of poisoning in Iran.

Drug poisoning has also been reported as the most common cause of poisoning in other studies (31). Among the causes of poisoning, narcotics and painkillers accounted for the largest share (33). The most common cause of poisoning in the United States was analgesic medication poisoning, including acetaminophen (34). While in our study opium poisoning was the most prevalent.

Access to painkillers as over-the-counter medicine is easy and acetaminophen is responsible for a significantly higher rate of poisoning in comparison to other overthe-counter (OTC) medications like NSAIDs. Also, the ranking of medications in our study shows that OTCmedication or non-OTC-medication does not seem to be responsible for the rate of consumption. This is a warning for health policymakers regarding the management of the drugs and medication available to the public; while many other factors may have an effect on our results as well as intentional or accidental poisoning.

The main issue investigated in the present study is the identification of the causes of toxicity in cases of intentional poisoning or suicidal attempts. With the increasing prescription of antidepressants in many countries, suicide rates have increased. Studies have shown that with the increased rate of the prescription of antidepressants; while being effective in controlling depression as one of the most important risk factors of suicide, they are causing an increased incidence of poisoning through suicide or drug overdose. However, in some countries increased prescription of the antidepressants was beneficial $(35,36)$. Findings showed that benzodiazepines were the second agent responsible for the poisoning in Iran. But the results regarding the TCA and SSRIs are contradictory as those are ranked as the least common causes of poisoning. One reason elucidating this issue could be due to the fact that benzodiazepines like diazepam are better known by the Iranian people than TCA or SSRI. Also, the low rank of TCA and SSRI toxicity in Iran may happen because of a 
higher prescription rate of benzodiazepines in comparison to the TCA and SSRIs. As a result, it is necessary to design and present an appropriate training program regarding the considerations of how to prescribe medicine in medical education. In prescribing medications, the patient's mental state, and the number of pills prescribed should be taken into account.

There are shreds of evidence regarding the effect of the type of medications available in the market on the suicide pattern. For example, in Sweden, the replacement of SSRIs instead of TCAs was effective in the prevention of SSRIs poisoning but increased the benzodiazepine poisoning rate (37).

In our study, the average death rate was $5.39 \%$. Rapid access to medical centers for poisoning can justify low mortality rates. In most cases, hydration, gastric lavage, activated charcoal, antidotes, and laxatives are being used to treat the poisoned patients. There are often issues regarding the management of the poisoning cases in the emergency department as well as inadequate knowledge of physicians dealing with the poisoned patient (38).

Also, there are concerns about the high incidence of organophosphate and aluminum phosphate poisoning. In this regard, it is suggested that public education be conducted to increase the level of public awareness through mass media to prevent drug poisoning.

The findings of this study are limited in generalizability due to various factors like age, sex, culture, etc. The substance used for intentional poisoning is related to various factors. Thus, age, sex, place of birth, and access to the substance of poisoning have a profound effect on this option in attempting suicide.

\section{Conclusion}

Due to several influential factors, further research is needed to implant preventive public policies on the education of the people or market availability of medications. Our study has been conducted on a national scale without any time constraints. Although the generalizability of the results may be influenced by the mentioned confounding factors, the results of this study provide a viewpoint on the cause of the poisoning in the country as we have provided a ranking of the most possible poisoning etiologies that may refer to the emergency department and this would be useful for physicians in cases of poisoning with unknown sources.

\section{Authors contributions}

The study was designed by MF, AH, and SD. The search strategy protocol was determined by EB, MF, BS and $\mathrm{ZH}$. Searching was conducted by SA, SD, and RA. RA and BS did the analysis. All authors contributed in draft preparation and its revisions.

\section{Ethical issues}

All sources used to conduct this study were rederived based on the permissions of research authors in sharing the data.

\section{Supplementary Materials}

Supplementary file 1 contains Figure S1 and Table S1.

\section{References}

1. Jones LO. Poisoning. Anaesthesia \& Intensive Care Medicine. 2006; 7(4): 132-4.

2. Assar S, Hatami S, Lak E, Pipelzadeh M, Joorabian M. Acute poisoning in children. Pak J Med Sci 2009; 25(1): 51-4.

3. Krenzelok EP, Dunmire SM. Acute poisoning emergencies. Resolving the gastric decontamination controversy. Postgrad Med 1992; 91(2): 179-82, 85-6. doi: 10.1080/00325481.1992.11701204.

4. Mehrpour O, Akbari A, Jahani F, Amirabadizadeh A, Allahyari E, Mansouri B, et al. Epidemiological and clinical profiles of acute poisoning in patients admitted to the intensive care unit in eastern Iran (2010 to 2017). BMC Emerg Med 2018; 18(1): 30. doi: 10.1186/s12873-018-01816.

5. Yardley R, Chandrashekaraiah S. Non-traumatic brain injuries. In: Arora N, Laha SK, eds. The Beginner's Guide to Intensive Care. CRC Press; 2018.

6. Chrysanthopoulou E, Karampela I, Patsilinakou S, Konstantopoulou G, Franzeskaki F, Kontogiorgi M, et al. Admission to intensive care unit due to non-traumatic coma: etiology and outcome. Eur Respir J 2019; 54(Suppl 63): PA2282. doi: 10.1183/13993003.congress-2019. PA2282.

7. Stanca S, Ulmeanu CE, Stanca HT, Iovanescu G. Clinical features in toxic coma in children. Exp Ther Med 2019; 18(6): 5082-7. doi: 10.3892/etm.2019.7857.

8. McCoy M, Stephens-Stidham S, Barnard J, Williams B. Unintentional Drug Poisoning Deaths in Dallas County from 1997 to 2017. In: APHA's 2019 Annual Meeting and Expo; November 2-6, 2019; Philadelphia, USA.

9. Kim M, Shin SD, Jeong S, Kwak YH, Suh GJ. Poisoninginduced Out-of-Hospital Cardiac Arrest and Outcomes according to Poison Agent. J Korean Med Sci 2017; 32(12): 2042-50. doi: 10.3346/jkms.2017.32.12.2042.

10. Alzahrani SH, Alqahtani AH, Farahat FM, Elnour MAG, Bashawri J. Drug poisoning and associated factors in western Saudi Arabia: a five-year retrospective chart review (2011-2016). Pak J Med Sci 2017; 33(5): 1188-93. doi: 10.12669/pjms.335.13119.

11. Singh O, Nasa P, Juneja D. General poisoning management. In: Chawla R, Todi S, eds. ICU Protocols. Singapore: Springer; 2020. p. 151-8. doi: 10.1007/978-981-15-09025_14.

12. Afshari R, Majdzadeh R, Balali-Mood M. Pattern of acute poisonings in Mashhad, Iran 1993-2000. J Toxicol Clin Toxicol 2004; 42(7): 965-75. doi: 10.1081/clt-200042550.

13. Hassanian-Moghaddam H, Pajoumand A, Sarjami S. One year epidemiological study of acute adult and adolescent poisoning admitted to Loghman hospital, Tehran. 20042005. Scientific Journal of Forensic Medicine 2008; 13(4): 235-40. [In Persian].

14. Sheikhazadi A, Saberi Anary SH, Ghadyani MH. Nonfire carbon monoxide-related deaths: a survey in Tehran, Iran 
(2002-2006). Am J Forensic Med Pathol 2010; 31(4): 35963. doi: 10.1097/PAF.0b013e3181f23e02.

15. Dianat I, Nazari J. Characteristics of unintentional carbon monoxide poisoning in Northwest Iran--Tabriz. Int J Inj Contr Saf Promot 2011; 18(4): 313-20. doi: 10.1080/17457300.2011.589006.

16. Islambulchilar $\mathrm{M}$, Islambulchilar Z, Kargar-Maher $\mathrm{MH}$. Acute adult poisoning cases admitted to a university hospital in Tabriz, Iran. Hum Exp Toxicol 2009; 28(4): 18590. doi: 10.1177/0960327108099679.

17. Sobhani AR, Shojaii-Tehrani H, Nikpour E, Noroozi-Rad $\mathrm{N}$. Drug and chemical poisoning in northern Iran. Arch Iran Med. 2000;3(2):1-4.

18. Soltaninejad K. Outbreak of mushroom poisoning in Iran: April-May, 2018. Int J Occup Environ Med 2018; 9(3): 1526. doi: 10.15171/ijoem.2018.1380.

19. Shokrzadeh M, Hajimohammadi A, Hoseinpoor R, Delaram A, Shayeste Y. An epidemiological survey of drug poisoning and a comparison with other poisonings cases admitted to a university hospital in Gorgan, Iran, 20082015. Int J Epidemiol Res 2017; 4(2): 94-103.

20. Feiz Disfani H, Kamandi M, Mousavi SM, Sadrzadeh SM, Farzaneh R, Doolabi N, et al. Risk factors contributing to the incidence and mortality of acute childhood poisoning in emergency department patients in Iran: a hospital-based casecontrol study. Epidemiol Health 2019; 41: e2019016. doi: 10.4178/epih.e2019016.

21. Jafarzadeh S, Khiyali Z, Safdar S, Mobasheri F, Maleki Z. Characteristics of hospitalized patients due to intentional and unintentional poisoning in Fasa city, 2014-2016. Iranian Journal of Emergency Care 2017; 1(2): 9-18. [Persian].

22. Eslami M, Kousha S, Fesharaki M, Kazemi N, Ghafarzad A, Zamani M, et al. Evaluation prevalence and causes of drug and chemical poisoning in patients referred to emergency wards of Sina hospital of Tabriz University of Medical Sciences at 2012-13. Int J Curr Res Acad Rev 2014; 2(7): 187-94.

23. Masoumi G, Ganjei Z, Teymoori E, Sabzghabaee AM, Yaraghi A, Akabri M, et al. Evaluating the prevalence of intentional and unintentional poisoning in vulnerable patients admitted to a referral hospital. Journal of Isfahan Medical School 2013; 31(252): 1452-60. [In Persian].

24. Afzali S, Mani Kashani K, Abbasi Kolsoum F. Pattern of mortality due to poisoning by drugs and chemical agents in Hamadan, Iran, 2005-2007. Qom University of Medical Sciences Journal. 2008; 2(2): 27-32. [In Persian].

25. Ahmadi H, Hosseini J, Rezaei M. Epidemiology of tramadol overdose in Imam Khomeini hospital, Kermanshah, Iran (2008). Journal of Kermanshah University of Medical Sciences 2011; 15(1): 72-7. [In Persian].

26. Hashemnejad M, Fatehi R. Epidemiological study of poisoning in patients of Karaj Shariati hospital in 2011 to 2012. Int J Med Toxicol Forensic Med 2014; 4(1): 17-22.

27. Torkashvand F, Sheikh Fathollahi M, Shamsi S, Kamali M,
Rezaeian M. Evaluating the pattern of acute poisoning in cases referred to the emergency department of Ali-ebn Abi Taleb hospital of Rafsanjan from October 2013 to September 2014. Journal of Rafsanjan University of Medical Sciences 2015; 14(4): 311-24. [In Persian].

28. Sadeghieh Ahari S, Farzaneh E, Amani F, Azari SH. Epidemiology of poisoning due to agricultural pesticides in patients referred to Ardabil city hospitals, 2012. Journal of Health and Hygiene 2014; 5(3): 240-7. [In Persian].

29. Najari F, Dadpour B, Baradaran Kayal I, Khalilzadeh M. Evaluation of the patterns of mortality due to acute poisoning in Imam Reza hospital, Mashhad, Iran, 2016. J Toxicol Cur Res 2017; 1: 001. doi: 10.24966/tcr-3735/100001.

30. Dehghani R, Fathi B, Aboo-Saaidi Z, Jalalati A, Ramezani M, Nohi M. Epidemiology of poisonings in Shahid Beheshti hospital in Kashan, Iran. Int J Med Toxicol Forensic Med 2015; 5(3): 144-50. doi: 10.22037/ijmtfm. v5i3(Summer).7578.

31. Azekour K, Belamalem S, Soulaymani A, El Houate B, El Bouhali B. Epidemiological profile of drug overdose reported in South-East Morocco from 2004 to 2016. Drugs Real World Outcomes 2019; 6(1): 11-7. doi: 10.1007/ s40801-019-0148-2.

32. Haghgou H. Suicide in Tehran, 1969-1974. Tehran: Tehran University, Faculty of Social Silences, institute for Social studies: 1997 . [In Persian].

33. Iwersen-Bergmann S, Andresen-Streichert H. [Poisoning by addictive substances, laboratory tests, and forensic medical death clarification in the case of poisoning]. Bundesgesundheitsblatt Gesundheitsforschung Gesundheitsschutz 2019; 62(11): 1362-9. doi: 10.1007/ s00103-019-03027-4.

34. Nourjah P, Ahmad SR, Karwoski C, Willy M. Estimates of acetaminophen (Paracetomal)-associated overdoses in the United States. Pharmacoepidemiol Drug Saf 2006; 15(6): 398-405. doi: 10.1002/pds.1191.

35. Isacsson G, Rich CL, Jureidini J, Raven M. The increased use of antidepressants has contributed to the worldwide reduction in suicide rates. Br J Psychiatry 2010; 196(6): 42933. doi: 10.1192/bjp.bp.109.076166.

36. Korkeila J, Salminen JK, Hiekkanen H, Salokangas RK. Use of antidepressants and suicide rate in Finland: an ecological study. J Clin Psychiatry 2007; 68(4): 505-11. doi: 10.4088/ jcp.v68n0403.

37. Zahl PH, De Leo D, Ekeberg $\varnothing$, Hjelmeland H, Dieserud G. The relationship between sales of SSRI, TCA and suicide rates in the Nordic countries. BMC Psychiatry 2010; 10: 62. doi: 10.1186/1471-244x-10-62.

38. Yaraghi A, Eizadi-Mood N, Gheshlaghi F, Montazeri K, Memarzadeh MA, Massoumi GR, et al. Evaluation of the therapeutic interventions performed in patients before and during referral to poisoning emergency department in Isfahan province. Scientific Journal of Forensic Medicine 2014; 19(1-4): 343-9. [In Persian]. 